\title{
Disconnective surgery in posterior quadrantic epilepsy: experience in a consecutive series of 10 patients
}

\author{
Christian Dorfer, M.D., ${ }^{1}$ Thomas Czech, M.D., ${ }^{1}$ \\ Angelika Mühlebner-Fahrngruber, M.D., ${ }^{2}$ Aygül Mert, M.D., ${ }^{1}$ Gudrun Gröppel, M.D., ${ }^{2}$ \\ Klaus Novak, M.D., ${ }^{1}$ Anastasia Dressler, M.D., ${ }^{2}$ Edith Reiter-Fink, M.D., ${ }^{2}$ \\ Tatjana Traub-Weidinger, M.D., ${ }^{3}$ and Martha Feucht, M.D. ${ }^{2}$ \\ Departments of ${ }^{1}$ Neurosurgery, ${ }^{2}$ Pediatrics and Adolescence Medicine-Epilepsy Monitoring Unit, and \\ ${ }^{3}$ Nuclear Medicine, Medical University of Vienna, Austria
}

Object. Outcomes following functional hemispherotomy in patients with drug-resistant epilepsy have been well described. However, studies reporting long-term longitudinal outcomes after subhemispheric disconnective epilepsy surgery are still limited.

Methods. The authors conducted a retrospective review of prospectively collected data of 10 children who underwent temporoparietooccipital (TPO) disconnective surgery at the Vienna Pediatric Epilepsy Center.

Results. There were 3 males and 7 females (median age 8.7 years; range 4.2-22.1 years). The affected hemisphere was the left in 3 patients and the right in 7. The patients' median age at seizure onset was 3.0 years (range $0.2-8.3$ years). The median duration of epilepsy before surgery was 5.2 years (range 1.3-17.2 years). The underlying pathology was TPO malformation of cortical development in 5 patients, and venous infarction, posterior hemispheric quadrant atrophy, Sturge-Weber syndrome, cortical involvement of a systemic lupus erythematosus, and gliosis after cerebral tumor treatment in 1 each. In 6 children, a pure TPO disconnection was performed; in 2 patients, the temporal lobe was resected and parietooccipital disconnection was performed. The 2 remaining patients had had previous epilepsy surgery that was extended to a TPO disconnection: disconnection of the occipital lobe $(\mathrm{n}=1)$ and resection of the temporal lobe $(n=1)$. The authors encountered no complications while performing surgery. No patient needed blood replacement therapy. No patient developed CSF disturbances that warranted treatment. Nine of 10 patients are currently seizure free since surgery (Wieser Class 1a) at a median follow-up time of 2.1 years (range 4 months to 8.1 years).

Conclusions. Temporoparietooccipital disconnection is a safe and effective motor-sparing epilepsy surgery in selected cases. Technical adjuncts facilitate a better intraoperative visualization and orientation, thereby enabling a less invasive approach than previously suggested.

(http://thejns.org/doi/abs/10.3171/2013.3.FOCUS1362)

KEY WORDS $\quad$ posterior quadrantic epilepsy $\bullet \quad$ temporoparietooccipital surgery
3D surface navigation $\bullet \quad$ intraoperative monitoring $\bullet$ pediatric epilepsy surgery

$\mathrm{M}$ ULtilobar pathologies-making up 12\%-22\% of large epilepsy surgery series in children and $3 \%-9 \%$ of mixed pediatric/adult series -are not uncommon etiologies in early-onset drug-resistant epileptic encephalopathies. ${ }^{17}$ Children with intractable epilepsy due to extensive lesions involving the posterior quadrant (temporal, parietal, and occipital lobes) form a significant subset of this population. Early epilepsy sur-

Abbreviations used in this paper: $\mathrm{AED}=$ antiepileptic drug; $\mathrm{EEG}$ = electroencephalography; FDG = fluorine-18-labeled fluorodeoxyglucose; $\mathrm{MEP}=$ motor evoked potential; $\mathrm{PO}=$ parietoocipital; $\mathrm{SEP}$ $=$ somatosensory evoked potential; TPO = temporoparietooccipital. gery is the treatment of choice in most of these patients, but conventional resective surgery carries substantial operative risks.

Similar to the continuous evolution of disconnective techniques in functional hemispherectomy, the surgical approach for TPO epilepsies has evolved progressively toward more disconnection and less resection. ${ }^{5,6,18,20,21}$

Disconnective procedures are based on the concept that interrupting the epileptic discharge-spreading pathway and isolating the primary epileptogenic zone would have the same effect as removing the epileptic focus. The potential benefits of this approach include smaller craniotomies, reduced blood loss, and fewer long-term compli- 
cations, such as hemosiderosis and disturbances of CSF circulation, without minimizing the effect on postoperative seizure outcome. On the other hand, disconnective surgery can theoretically run the risk of leaving the epileptogenic cortex connected to the rest of the brain due to complicated intraoperative orientation.

New techniques, including 3D surface navigation and intraoperative monitoring, further minimize brain exposure and facilitate a safe and complete disconnection based on a precise understanding of the relevant anatomy.

We present our experience using these techniques in our series of TPO disconnections.

\section{Methods}

All patients who had TPO disconnections at the Vienna Pediatric Epilepsy Center were evaluated retrospectively (data collection, however, was prospective). Approval from the Medical University of Vienna Clinical Research Ethics Board was obtained.

\section{Presurgical Evaluation}

Presurgical evaluation followed a standardized protocol, ${ }^{10,14}$ including thorough neurological, ophthalmological, neuropsychological, and psychiatric assessments, as well as intensive video-EEG monitoring and highresolution MRI. ${ }^{19}$ Also, FDG-PET was performed in all patients to evaluate the functional status of the frontocentral lobe and the contralateral hemisphere..$^{13}$ In an attempt to define more precisely the extent of dysplasia and/or to detect dysplastic cortex in the contralateral hemisphere, ${ }^{12}$ C-methionine PET was performed. ${ }^{2}$ If necessary, invasive recordings and stimulation of eloquent cortical areas were also performed.

Before surgery, well-tolerated AED monotherapy was established.

\section{Surgical Technique}

Patients were placed supine with the ipsilateral shoulder elevated. The head was fixed in a 3-pin Mayfield system and turned to the opposite side. With the use of a navigation system, a straight or slightly $\mathrm{S}$-shaped skin incision was made from the anterior tragus across $2 \mathrm{~cm}$ to the midline in the postcentral area. An elongated bone flap was cut, exposing the postcentral region from the midline down to the posterior temporal operculum (Fig. 1 left). After confirming the location of the central sulcus by median nerve SEP phase reversal recorded through a
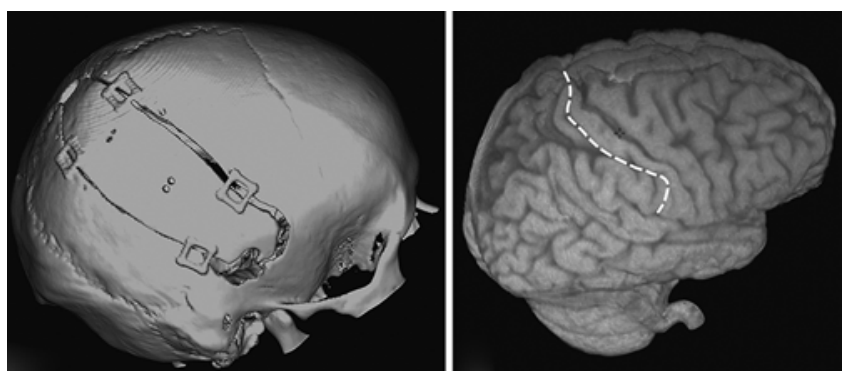

FIG. 1. Left: Postoperative 3D reconstructed CT scan showing the tailored craniotomy. Right: 3D surface rendering with the disconnection line along the postcentral sulcus. subdural grid electrode, an 8-contact subdural strip electrode was positioned on the long axis of the postcentral gyrus. Thereby, continuous SEP monitoring during the disconnection procedure was provided. In case of a disconnection along the central sulcus, MEPs were monitored by direct stimulation through a subdural grid electrode positioned on the motor strip.

Disconnections were started with a corticotomy and subpial suction/resection at the parietal operculum reaching the ventricular trigone. In cases with small ventricles, the trigone was punctured through the low parietal cortex with the help of the navigation. The corticotomy along the anterior incision line was connected to the ventricle. The medial cortex was resected subpially down to the corpus callosum. The callosotomy from the posterior isthmic part through the splenium was performed in a subpial fashion until we achieved visualization of the galenic vein complex through the pia/arachnoid layer. The fornix was then cut at the level of the trigone. After subpial resection of the parietotemporal opercular cortex down to the posterior corner of the insula, we subpially resected the upper surface of T1 along the inferior insular sulcus. The incision through the white matter of the temporal stem followed the temporal horn to the anterior end of the choroid fissure. The disconnection was completed by suction/dissection through the dorsal part of the amygdala subpially following the lower border of the middle cerebral artery (Figs. 1 right; 2 and 3).

Large crossing veins, arteries, and bridging veins were spared throughout the procedure. After thoroughly rinsing the ventricular system with synthetic CSF, the disconnection line was sealed with Lyostypt (Aesculap, Braun) and fibrin glue (Baxter). No drainage was used.

All procedures were performed by the same neurosurgeon (T.C.).

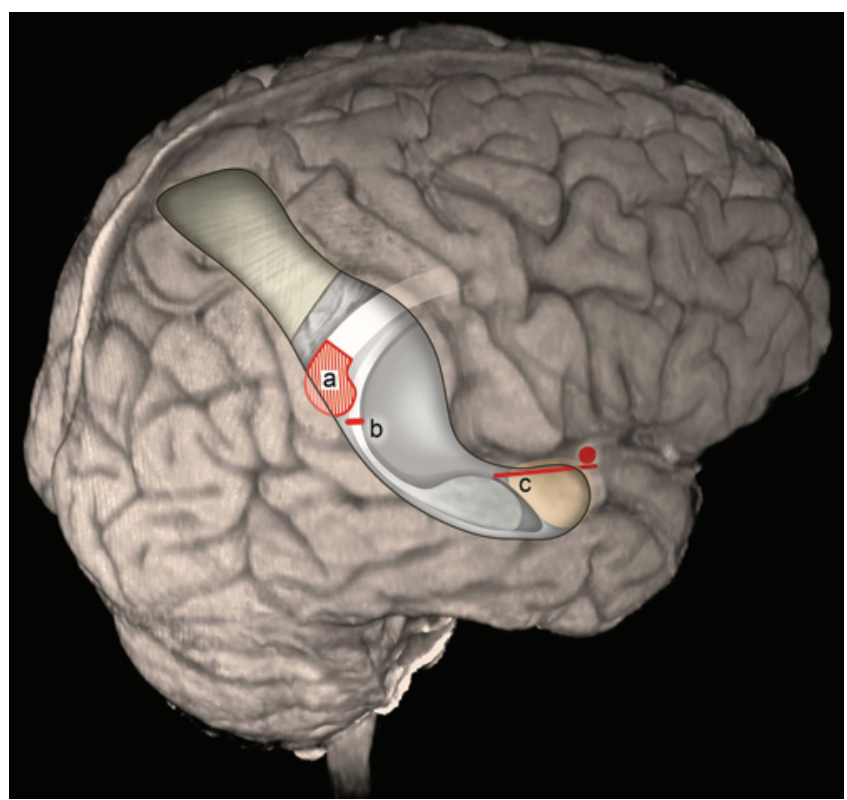

FIG. 2. Semischematic view of the procedural approach; the corticotomy window has been enlarged to better illustrate the relevant anatomy: section of the splenium (a), section of the fornix behind the pulvinar (b), and disconnection line from the anterior choroidal point through the upper part of the amygdala (c). 

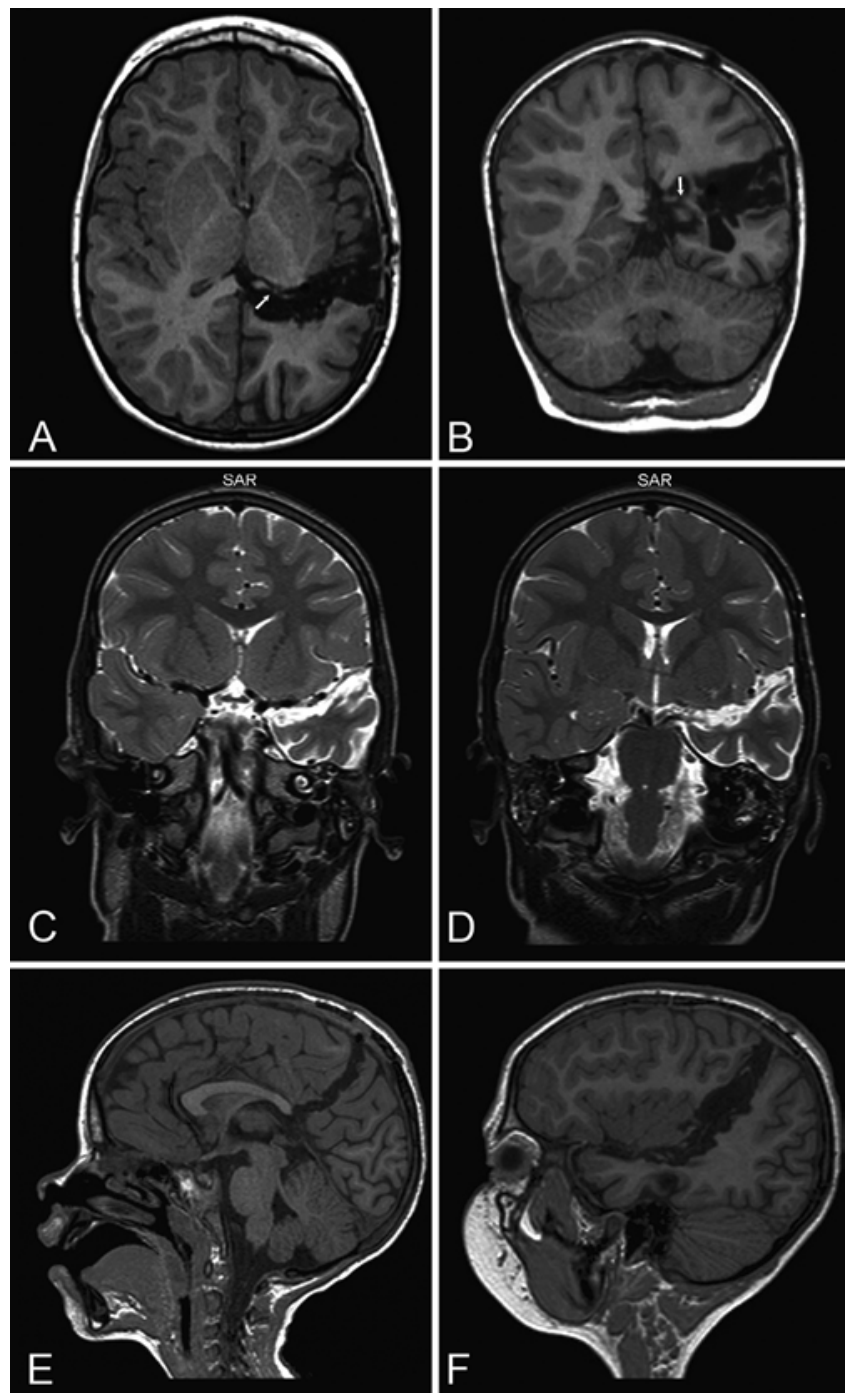

FIG. 3. Postoperative MR images illustrating the section of the fornix (arrow, $\mathbf{A}$ and $\mathbf{B}$ ), the disconnection of the temporal lobe (C and D), the medial disconnection line with section of the splenium (E), and the disconnection line along the inferior insular sulcus through the temporal stem (F)

\section{D Surface Navigation}

Neuronavigation was used in all cases (Fig. 1). Direct intraoperative navigation of the 3D brain surface was additionally performed in the last 3 patients of our series by the use of Synergy Cranial version 2.2 software of the commercially available neuronavigation system S7 (Medtronic), which was made available in September 2011. For brain volume extraction, the semi-automated brain volume extraction tool included in the Synergy Cranial navigation software was used. It is based on a "watershed" algorithm that builds a brain volume based on the signal threshold between cortex and cancellous bone on MR images. ${ }^{16}$ Depending on the individual MR scanner and acquisition, threshold values have to be adjusted manually (our settings: lower threshold 200; upper threshold 1200; filter, medium). The data for creating the 3D brain models was provided by T1-weighted MR images with and without contrast enhancement. The direct volume rendering module of the navigation software was used for intraoperative display of the 3D brain model. For transparency of the 3D brain surface, we selected the predefined transfer function "basic volume rendering."

\section{Intraoperative Neurophysiology}

Intraoperative neurophysiology included techniques of neurophysiological mapping and monitoring. After bilateral median nerve and tibial nerve stimulation, SEPs were recorded from scalp electrodes. Motor evoked potentials using transcranial electrical high-voltage stimulation were recorded from limb muscles of the upper and lower extremities. Baseline values of MEPs were established using short trains of transcranial stimuli, stimulus duration of $500 \mu \mathrm{sec}$, interstimulus interval of $4 \mathrm{msec}$, and intensity of 40-180 mA. Baseline recordings of median nerve and tibial nerve SEPs were established before a subdural electrode array (4- or 8-contact strip electrode or 20-contact grid electrode) was placed on the central area cortical surface for the functional localization of the central sulcus using the SEP phase reversal technique. To assess the functional anatomy of the central area, results of a phase reversal technique were correlated to a surface rendering of preoperative MRI. Consecutively, using the subdural electrode array, the primary motor area was mapped for best motor response of MEPs elicited by direct cortical stimulation. At least single-muscle response from contralateral upper or lower extremity at lowest stimulus intensity was established for continuous monitoring of motor pathways' functional integrity. Short trains of 5-7 stimuli, duration of $500 \mu \mathrm{sec}$, and intensity of 5-25 mA were applied via a subdural electrode contact. In individual patients, mapping of cortically and subcortically elicited MEPs was performed repeatedly during the surgical procedure to verify the topography of the primary motor cortex and localization of corticospinal tract fibers in relation to the line of white matter disconnection. Additionally, in some patients, SEPs were recorded from the 20-contact grid electrode or from an additionally placed strip electrode.

\section{Postoperative Follow-Up}

Outcome was assessed on an inpatient basis 3 months after surgery, once per year for 5 years, and every 2 years from then on if stable. Patients were seen more frequently if problems occurred.

Postoperative examinations included thorough neurological, neuropsychological, neuroophthalmological, and psychiatric assessments, as well as 48-hour video-EEG monitoring. Magnetic resonance imaging was performed at 3 months, 12 months, and 5 years after surgery. Seizure outcome was classified based on patient and family reporting, according to the International League of Epilepsy proposal. ${ }^{22}$

Withdrawal of AEDs was recommended 2 years after surgery, provided that the patient was completely seizure free and EEG control examinations did not show epileptic activity. 


\section{Dorfer et al.}

\section{Results}

\section{Preoperative Details}

Of the 238 children with drug-resistant epilepsy who had had epilepsy surgery at our center, 10 (4.2\%) underwent posterior quadrantic disconnective surgery. There were 3 boys and 7 girls. The affected hemisphere was the left in 3 patients and the right in 7 patients. The median age at seizure onset was 3.0 years (range $0.2-8.3$ years). The median duration of epilepsy before surgery was 5.2 years (range 1.3-17.2 years). The median age at surgery was 8.7 years (range 4.2-22.1 years). Seizure frequency before surgery was high in all patients; seizures occurred on a daily basis in $60 \%$ of the patients. Table 1 summarizes the patients' clinical characteristics.

The underlying pathology was malformation of cortical development in 5 patients $(50 \%)$. Venous infarction, posterior hemispheric quadrant atrophy, Sturge-Weber syndrome, cortical involvement of a systemic lupus erythematosus, and gliosis after cerebral tumor treatment were the underlying pathologies in 1 patient each.

One patient (Case 9) had had previous brain surgeries with resection of a trigonal plexus carcinoma and subsequent implantation of a ventriculoperitoneal shunt and an Ommaya reservoir.

Preoperative neurological examination revealed a slight degree of hemiparesis in 4 patients, but fine finger movements and foot tapping were possible in all. All patients were ambulatory.

In all patients, FDG-PET confirmed the functional integrity of the contralateral hemisphere. In the patient (Case 6) with Sturge-Weber syndrome, the whole affected hemisphere, including the frontocentral region, showed homogeneous decrease of tracer uptake compared with the contralateral hemisphere.

Complete hemianopia was present in 3 patients and incomplete hemianopia in 1 . No visual field abnormalities were identified in the rest of the patients, although young age and/or mental retardation made clinical assessment less reliable.

\section{Operative Details}

In 6 patients, a pure TPO disconnection was performed. In 2 patients, temporal lobectomy was combined with PO disconnection.

Two patients had had previous epilepsy surgery (with an unfavorable seizure outcome) that was extended to a TPO disconnection: the patient (Case 6) with Sturge-Weber syndrome had a prior disconnection of the occipital lobe that had led to a less than $50 \%$ reduction in seizure frequency; the patient in Case 10 had undergone a temporal lobectomy, was seizure free for 2 years, but eventually experienced seizure recurrence. In this patient, invasive monitoring with subdural grids and depth electrodes was subsequently performed to evaluate if a more focal cortical resection in the $\mathrm{PO}$ region rather than a complete $\mathrm{PO}$ disconnection should be performed.

We encountered no serious intraoperative complications. No patient needed blood replacement. No patient had a CSF disturbance that warranted treatment.

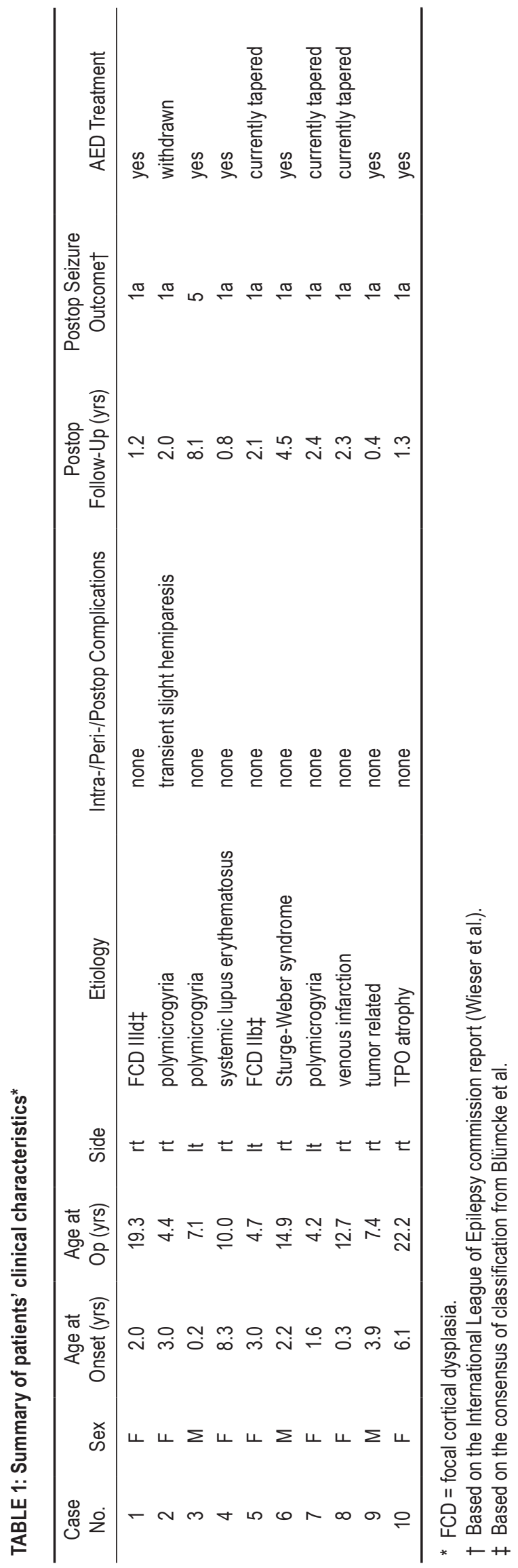

Neurosurg Focus / Volume 34 / June 2013 


\section{Disconnective surgery in posterior quadrantic epilepsy}

\section{Postoperative Outcome}

No patient was lost to follow-up.

As expected, all patients had complete hemianopia after surgery. Only 1 patient had a slight de novo motor deficit that fully recovered after 6 months.

Nine of 10 patients are currently seizure free without auras (Wieser Class 1a) at a median follow-up time of 2.1 years (range 4 months to 8.8 years). ${ }^{22}$ One patient was seizure free until AED reduction caused seizure relapse, but seizures have been controlled with medication again for 3 years. Recently, however, the seizures returned (Wieser Class 5). Histology in this patient had been classified as polymicrogyria and nodular heterotopia.

No patient has deteriorated (Wieser Class 6).

Antiepileptic drugs were completely withdrawn in 1 patient and are currently being gradually withdrawn in 3 patients.

\section{Discussion}

Multilobar involvement is not unusual in pediatric epilepsy patients. Thus, multilobar resection can be considered in medically refractory patients after presurgical evaluation in selected cases. ${ }^{20}$

Posterior quadrant dysplasia has been described as a characteristic brain malformation. ${ }^{3}$ Surgical treatment of these posterior quadrantic epilepsies with involvement of the TPO area has also been performed in other medically refractory epilepsy syndromes. ${ }^{5,12}$

Similar to the evolution of disconnective techniques in hemispherectomy, multilobar disconnection rather than resection has been increasingly suggested in patients with posterior quadrantic epilepsy.,12 The potential benefits of this approach are the potential reduction of early and delayed complications such as blood loss, hemosiderosis, and disturbance of CSF circulation. On the other hand, disconnective surgery theoretically comprises the risk of leaving epileptogenic cortex "undisconnected" due to intraoperative orientation problems. This difficulty has been suggested to be a possible reason for unfavorable seizure outcome reported in some hemispherotomy series, especially in patients with malformation of cortical development.?

Completeness of the planned disconnection is essentially a function of the surgeon's experience with this type of surgery. Based on a precise understanding of the relevant anatomy, the technical adjuncts that a surgeon uses, including 3D surface navigation and intraoperative monitoring, facilitate a better intraoperative orientation and identification of essential cortical areas, thereby enabling a safe and complete disconnection.

So far, only a few studies have focused on posterior quadrantic epilepsy surgery. $3,5,8,9,12,19$ In these studies, identification of the central cortical areas was based on macroscopic examination of the gyral pattern and correlation of these findings with preoperative $3 \mathrm{D}$ reconstructed MR images. Localization of the cortical surface in relation to the gyral and sulcal architecture is problematic, as anatomical variability is high. ${ }^{15}$ The neuronavigational system with the $2 \mathrm{D}$ images in axial, coronal, and sagittal planes was deemed to be less helpful.,12 The reason for this might be that creating a 3D mental image of the brain from the $2 \mathrm{D}$ images is difficult and might lead to misinterpretation and incorrect localization. Technical advances now allow reconstruction of the 2D image data set into a 3D display on the navigational system. The resultant 3D images, including those of surface veins, have been shown to be useful for intraoperative orientation independent from brain shift in cerebral tumor surgery. ${ }^{11}$

We are now using this application in all epilepsy surgery cases to guarantee correct identification of the gyral pattern. Applying this technology in TPO surgery has the advantage of reducing the area of brain exposure (that is, performing a more tailored approach).

Previous series that relied on anatomical patterns reported that a large exposure of the cortex is essential to be able to identify the central cortical areas and to correctly define the disconnection line. ${ }^{5,12}$ With this extensive approach, surgery was a major and long procedure that could have resulted in considerable blood loss and fluid shifts. This may be associated with hypovolemia and death, especially in infants. A more tailored approach for TPO surgery exposes just the cortical areas essential for performing the disconnection, obviating the need to expose the brain for identification purposes. This is well accomplished by the 3D surface navigation and certainly represents the latest evolution of operative approaches aiming to be less invasive. ${ }^{5}$

Relying solely on the morphology to define functional areas in the central region might be misleading. For this reason, we applied electrophysiological measures to localize the sensorimotor functional cortex with certainty. The importance of functional cortical mapping in TPO surgery has already been pointed out. ${ }^{5}$

In addition to using electrophysiology for cortical mapping, continuous monitoring of the primary sensory or motor pathways can be used throughout the disconnection procedure. We performed continuous cortical SEP and/or MEP monitoring to optimize the planned disconnection and to increase the safety of the procedure. This in turn maximizes the extent of disconnection and safety of surgery.

Published data about seizure outcome of posterior quadrantic epilepsy surgery are difficult to interpret because there are only a few studies that focus on this subgroup of epilepsy. ${ }^{3-5,8,9,12,17}$ Seizure outcome in these series differs significantly, with seizure freedom ranging from $50 \%$ to $92 \%$. This variety is due to differences in patient selection and surgical techniques.

If presurgical evaluation shows congruent data from MRI, PET, interictal/ictal scalp EEG, and clinical neurological and neuropsychological examinations indicating seizure onset in one posterior quadrant favorable, seizure outcome can be expected. . $^{3,5}$

A recently published study reported by Mohamed et al. ${ }^{12}$ has also included children with normal or nonlocalizing MRI findings and with more generalized seizure patterns on EEG. Six of the 16 children had generalized hypsarrhythmia on scalp EEG and 4 children had a typical EEG patterns seen in Lennox-Gastaut syndrome. Seizure outcome after TPO surgery was Engel Class I in only $56 \%$ of the patients. 


\section{Conclusions}

Temporoparietooccipital disconnection is a safe and effective motor-sparing epilepsy surgery in selected patients. Technical adjuncts, including 3D surface navigation and intraoperative electrophysiological monitoring, facilitate a better visualization and orientation, enabling a less invasive approach than previously suggested.

\section{Disclosure}

The authors report no conflict of interest concerning the materials or methods used in this study or the findings specified in this paper.

Author contributions to the study and manuscript preparation include the following. Conception and design: Czech, Dorfer, Feucht. Acquisition of data: all authors. Analysis and interpretation of data: Czech, Dorfer, Mühlebner-Fahrngruber, Gröppel, Dressler, Reiter-Fink, Traub-Weidinger, Feucht. Drafting the article: Czech, Dorfer. Critically revising the article: Czech, Dorfer, Novak, Feucht. Reviewed submitted version of manuscript: Czech, Dorfer, Feucht. Approved the final version of the manuscript on behalf of all authors: Czech. Administrative/technical/material support: Czech, Dorfer, Mühlebner-Fahrngruber, Mert, Gröppel, Novak, Traub-Weidinger. Study supervision: Czech, Feucht.

\section{References}

1. Blümcke I, Thorn M, Aronica E, Armstrong DD, Vinters HV, Palmini A, et al: The clinicopathologic spectrum of focal cortical dysplasias: a consensus classification purposed by an ad hoc Task Force of the ILAE Diagnostic Methods Commission. Epilepsia 52:158-174, 2011

2. Caplan R, Chugani HT, Messa C, Guthrie D, Sigman M, de Traversay J, et al: Hemispherectomy for intractable seizures: presurgical cerebral glucose metabolism and post-surgical non-verbal communication. Dev Med Child Neurol 35:582592,1995

3. D’Agostino MD, Bastos A, Piras C, Bernasconi A, Grisar T, Tsur VG, et al: Posterior quadrantic dysplasia or hemi-hemimegalencephaly: a characteristic brain malformation. Neurology 62:2214-2220, 2004

4. Dalmagro CL, Bianchin MM, Velasco TR, Alexandre V Jr, Walz R, Terra-Bustamante VC, et al: Clinical features of patients with posterior cortex epilepsies and predictors of surgical outcome. Epilepsia 46:1442-1449, 2005

5. Daniel RT, Meagher-Villemure K, Farmer JP, Andermann F, Villemure JG: Posterior quadrantic epilepsy surgery: technical variants, surgical anatomy, and case series. Epilepsia 48: 1429-1437, 2007

6. Delalande O, Bulteau C, Dellatolas G, Fohlen M, Jalin C, Buret $\mathrm{V}$, et al: Vertical parasagittal hemispherotomy: surgical procedures and clinical long-term outcomes in a population of 83 children. Neurosurgery 60 (2 Suppl 1):ONS19-ONS32, 2007

7. Devlin AM, Cross JH, Harkness W, Chong WK, Harding B, Vargha-Khadem F, et al: Clinical outcomes of hemispherectomy for epilepsy in childhood and adolescence. Brain 126: 556-566, 2003

8. Fogarasi A, Boesebeck F, Tuxhorn I: A detailed analysis of symptomatic posterior cortex seizure semiology in children younger than seven years. Epilepsia 44:89-96, 2003

9. Leiphart JW, Peacock WJ, Mathern GW: Lobar and multilobar resections for medically intractable pediatric epilepsy. Pediatr Neurosurg 34:311-318, 2001

10. Mayer H, Benninger F, Urak L, Plattner B, Geldner J, Feucht M: EKG abnormalities in children and adolescents with symptomatic temporal lobe epilepsy. Neurology 63:324-328, 2004

11. Mert A, Buehler K, Sutherland GR, Tomanek B, Widhalm G, Kasprian G, et al: Brain tumor surgery with 3-dimensional surface navigation. Neurosurgery 71 (2 Suppl Operative): ons286-ons295, 2012

12. Mohamed AR, Freeman JL, Maixner W, Bailey CA, Wrennall JA, Harvey AS: Temporoparietooccipital disconnection in children with intractable epilepsy. Clinical article. J Neurosurg Pediatr 7:660-670, 2011

13. Moosa AN, Gupta A, Jehi L, Marashly A, Cosmo G, Lachhwani D, et al: Longitudinal seizure outcome and prognostic predictors after hemispherectomy in 170 children. Neurology 80:253-260, 2013

14. Olbrich A, Urak L, Gröppel G, Serles W, Novak K, Porsche $\mathrm{B}$, et al: Semiology of temporal lobe epilepsy in children and adolescents. Value in lateralizing the seizure onset zone [corrected]. Epilepsy Res 48:103-110, 2002 (Erratum in Epilepsy Res 51:211, 2002)

15. Ribas GC: The cerebral sulci and gyri. Neurosurg Focus 28(2): $\mathrm{E} 2,2010$

16. Roerdink JB, Meijster A: The watershed transform: definitions, algorithms and parallelization strategies. Fundamenta Informaticae 41:187-228, 2000

17. Sarkis RA, Jehi LE, Najm IM, Kotagal P, Bingaman WE: Seizure outcomes following multilobar epilepsy surgery. Epilepsia 53:44-50, 2012

18. Schramm J, Kuczaty S, Sassen R, Elger CE, von Lehe M: Pediatric functional hemispherectomy: outcome in 92 patients. Acta Neurochir (Wien) 154:2017-2028, 2012

19. Sinclair DB, Wheatley M, Snyder T, Gross D, Ahmed N: Posterior resection for childhood epilepsy. Pediatr Neurol 32: 257-263, 2005

20. Villemure JG, Daniel RT: Peri-insular hemispherotomy in paediatric epilepsy. Childs Nerv Syst 22:967-981, 2006

21. Villemure JG, Peacock W: Multilobar resections and hemispherectomy, in Engel J Jr, Pedley TA (eds): Epilepsy: A Comprehensive Textbook. Philadelphia: Lippincott-Raven, 1997, pp 1829-1839

22. Wieser H, Blume W, Fish D, Goldensohn E, Hufnagel A, King $\mathrm{D}$, et al: ILAE commission report. Proposal for a new classification of outcome with respect to epiletic seizures following epilepsy surgery. Epilepsia 42:282-286, 2001

Manuscript submitted February 15, 2013.

Accepted March 19, 2013.

Please include this information when citing this paper: DOI: 10.3171/2013.3.FOCUS1362.

Address correspondence to: Thomas Czech, M.D., Department of Neurosurgery, Medical University of Vienna, Währinger Gürtel 18-20, 1090 Vienna, Austria. email: thomas.czech@meduniwien. ac.at. 